\title{
La tutoría en laparoscopia
}

\author{
Amón Sesmero JH. \\ Hospital Rio Hortega. Valladolid. \\ Actas Urol Esp 2006: 30 (5): 433-436
}

$\mathrm{L}^{\mathrm{a}}$ as técnicas laparoscópicas tienen cada vez más implantación en la práctica quirúrgica urológica. En este desarrollo ha jugado un papel predominante la urología europea. La puesta en marcha por equipos franceses de técnicas de laparoscopia pélvica como la prostatectomía radical, ha vuelto a definir el enfoque que se tenía sobre el techo de la laparoscopia urológica, ceñida a la patología renal y de la glándula suprarrenal desde que Clayman, en 1991, realizó la primera nefrectomía laparoscópica.

En poco tiempo se ha podido demostrar que las intervenciones laparoscópicas se pueden realizar en tiempos quirúrgicos superponibles a las técnicas abiertas deshaciendo con contundencia la crítica del escaso rendimiento con que se las asociaba. Si aspectos como la menor pérdida sanguínea y menor morbilidad, con la consiguiente disminución del postoperatorio y del tiempo de recuperación son evidentes, otros, concernientes al seguimiento oncológico, que necesitan un mayor tiempo para ser evaluados, no han frenado el entusiasmo con que la comunidad urológica se ha rendido a la laparoscopia.

En España, como ya manifesté hace algunos años, nos incorporamos a esta corriente de cambio con cierto retraso. Hace 5 años, mientras existía ya una importante actividad laparoscópica en otros países de nuestro entorno más próximo aquí la actividad era exigua ${ }^{1}$. Predominaba la idea de que la laparoscopia tenía un dudoso porvenir, que era un ejercicio técnico relegado a los pocos grupos que tradicionalmente la habían ejercitado, y que no merecía la pena hacer el esfuerzo de desarrollarla, dadas sus escasas indicaciones.

Basta echar un vistazo a las publicaciones sobre laparoscopia en nuestras revistas cientificas, o a la actividad comunicada en nuestros congresos antes de 2002 para comprobar que esta forma de cirugía era extraña para la mayoría.
Una evaluación posterior, sobre la actividad laparoscópica dentro de la $\mathrm{AEU}^{2}$, llevada a cabo en el segundo semestre de 2004, puso de manifiesto que nuestro desarrollo era equiparable al que tenian nuestros colegas de la Asociación Francesa de Urología hace 5 años, pero a pesar del evidente retraso, el análisis de esa actividad en los dos últimos años era ya un indicador del dinamismo que existe actualmente. Si hace algo más de una década la actividad estaba restringida a un pequeño número de hospitales, a partir de 2002 se incorporaron 24 servicios, y otro número importante de ellos tenian como proyecto iniciar esta actividad lo antes posible. En este momento son numerosos los equipos urológicos que están incorporando la laparoscopia a su labor quirúrgica.

Es indudable, pues, que existe un notorio interés en la aplicación de la laparoscopia, al tiempo que se evidencia una cada vez mayor demanda social. Definitivamente se ha comprendido que no se va a entender la práctica de la urología en un futuro cercano sin la laparoscopia, y, como ocurrió con la resección transuretral, su empleo se generalizará, pero ahora, a diferencia de lo que ocurrió con aquélla, se hará más rápidamente.

\section{Motivos del interés por la laparoscopia}

¿Qué ha motivado el interés actual?. Probablemente, uno de los aspectos que ha generado afán por incorporar la laparoscopia pueda ser su entrada en el campo oncológico. Aprender una técnica compleja para realizar una nefrectomía simple, de forma esporádica, o para reconstruir un riñón hidronefrótico, implicaba un esfuerzo difícilmente asimilable, pero cuando lo que vamos a tratar es un cáncer renal o prostático, -patología con la que nos enfrentamos diariamente- la perspectiva cambia. 
La capacidad de la laparoscopia para reproducir con rigor gran parte de las técnicas convencionales la convierte, no ya en una técnica particular para tratar una determinada patología, sino en una manera radicalmente nueva de enfocar gran parte del tratamiento quirúrgico de nuestra especialidad. Cuando apareció la litotricia extracorpórea por ondas de choque, la urología sufrió una pequeña revolución que afectaba al enfoque terapéutico de una parcela importante de su patología urológica, como era la litiasis. Entonces, gran parte de los urólogos -los que no disponían de un litotritor extracorpóreo- tuvieron la sensación de que perdían esa parcela, pero ahora esa "sensación de pérdida" puede ser mucho mayor, por lo que el urólogo ha asumido el reto de aprender y manejar la laparoscopia.

Otro matiz dinamizador es el desarrollo laparoscópico alcanzado por otras especialidades que pone en peligro la "responsabilidad" de la nuestra sobre ciertas patologías. Algunos cirujanos digestivos han comunicado la realización de nefrectomías y pieloplastias laparoscópicas, y la mayoría compiten con el urólogo por la patología suprarrenal. La sentencia de Clayman, "si no queremos hacerlo nosotros, otros lo harán" sintetiza la opinión de una gran parte de urólogos que han decidido aprender laparoscopia.

Pero quizás, un análisis más concreto, nos lleve a considerar que ha sido la realización de la prostatectomía radical por esta vía, la que ha conseguido más adeptos para la laparoscopia. La prostatectomía radical ha sido, a pesar de su dificultad, la intervención con la que muchos equipos han iniciado la laparoscopia, con lo que esto supone en cuanto a consideraciones éticas.

\section{Nivel de desarrollo actual}

Es indudable que existe una falta de correlación entre la cantidad de actividad laparoscópica puesta de manifiesto en publicaciones, comunicaciones a congresos, simposios, cursos con demostraciones de cirugía en vivo, etc. y el grado de implantación que realmente tiene. Incluso en el ámbito de países con avanzados sistemas de salud, como EEUU, Japón, Alemania o Inglaterra, la laparoscopia esta iniciando su desarrollo. Existen equipos veteranos que acu- mulan gran experiencia, pero no hay generalización de su empleo y no existe la posibilidad de tratar a todos los pacientes que se podrían beneficiar de una intervención laparoscópica y que son intervenidos por cirugía convencional.

Una encuesta realizada en el 2001, en el estado de California, sobre la práctica de la laparoscopia por diferentes especialidades ${ }^{3}$ puso de manifiesto que el $54 \%$ de los urólogos no la practicaban, y entre los practicantes, sólo un 5\% de ellos la realizaban de forma habitual en su práctica quirúrgica. Otra, de similares características, realizada en el 2003 y dirigida a 1.450 los urólogos de la sección Medio Oeste de la $\mathrm{AUA}^{4}$, revelaba que el $49 \%$ de los respondedores no realizaban laparoscopia y que en el $30 \%$ de los que la practicaban, representaba menos del 5\% de su actividad quirúrgica.

Ambas encuestas concluían denunciando el desarrollo incipiente y escaso de la laparoscopia en esos estados norteamericanos.

\section{Formación en laparoscopia}

La consecuencia lógica del interés por incorporar la laparoscopia como una práctica quirúrgica estandarizada, como antes comentábamos, es la creciente demanda de formación. Como recientemente explicaba en un editorial de European Urology Bertrand Guillonneau ${ }^{5}$, "existe premura en un gran número de alumnos en adquirir conocimiento de técnicas que precisan muchas horas de dedicación para ejercerlas con dominio".

Ahora bien, la formación laparoscópica es difícil y exige un sacrificio especial, sobre todo para el urólogo que ya está formado. El tópico de que no hay edad para aprender, no es aplicable en laparoscopia, ya que parece obvio que sí que la hay a la vista de las opiniones y actitudes de numerosos colegas que manifiestan que no van a "rentabilizar", debido a su edad, ese esfuerzo.

A pesar de ello, la mayor demanda actual de formación proviene de los urólogos ya formados, si bien, el desafio alcanza también la formación de las futuras promociones de residentes.

Estudios sobre el perfil del urólogo que desarrolla la laparoscopia ponen de manifiesto que la práctica es mayor en los urólogos formados durante el periodo de residencia (69\%) 
que los que lo han hecho como postgraduados e independiente de la intensidad con la que se haya llevado a cabo tal formación ${ }^{6}$.

Si queremos que la laparoscopia se generalice, necesitamos que las próximas generaciones de residentes se formen en ella, por lo que su entrenamiento en laparoscopia no debe descuidarse ya que puede ser la garantía de su posterior práctica. Esto significa que nos encontramos con la paradoja de tener que aprender al tiempo que debemos enseñar a nuestros residentes. Estamos en el momento del cambio, sobre nosotros pesa la responsabilidad de aprender, desarrollar y enseñar la laparoscopia.

\section{¿Cómo aprender laparoscopia?}

Cada vez es más frecuente el debate sobre ¿cómo debemos aprender?, ¿cómo debemos enseñar laparoscopia?. Respecto a la primera cuestión, hasta ahora ha sido la iniciativa individual la que ha emprendido el desafío del aprendizaje. La posibilidad, actualmente, de adquirir conocimientos teóricos es fácil gracias a la nutrida literatura científica y a la numerosa oferta de cursos, reuniones científicas y simposios urológicos en los que a través de cirugía "en directo" se puede ver realizar diferentes técnicas, sus indicaciones, resultados, complicaciones.

Esta etapa de impregnación, de conocimiento, de sensibilización es necesaria pero es obvio que la realización de la laparoscopia precisa de un nivel de formación elevado sustentado no sólo en unos amplios conocimientos teóricos, sino también en una habilidad técnica diferente a la quirúrgica tradicional, que precisa de un entrenamiento particularmente dirigido a desarrollar la coordinación ojo-mano al tener que operar mirando a un monitor que nos proyecta una imagen bidimensional, de la que, además, no tenemos percepción táctil.

Estas particulares características de la cirugía laparoscópica, hacen que el entrenamiento en pelvitrainer sea necesario para desarrollar este tipo de habilidad; entrenamiento que se sustentará en la repetición de gestos hasta conseguir la destreza y dominios suficientes en el desarrollo de la coordinación.

Ahora bien, se enfatiza demasiado en el desarrollo del aprendizaje laparoscópico mediante el entrenamiento en pelvitrainer físicos, y aunque es indudable que estos dispositivos sirven para desarrollar la coordinación, la destreza en gestos quirúrgicos como la sutura y el anudado a base de ejercicios prácticos repetidos, los ejercicios prácticos en vivo, utilizando el animal de experimentación aportan un mejor y más rápido aprendizaje ${ }^{7}$.

La cirugía experimental con cerdo (el modelo más adecuado para esta práctica) nos van a permitir una toma de contacto con lo que va a ser luego la cirugía en el paciente, en la realización de gestos y maniobras que precisan determinadas intervenciones y permite familiarizarnos con el instrumental laparoscópico. Estas fases del aprendizaje, aunque necesarias, son, además, insuficientes.

Se puede adquirir un grado de habilidad y rapidez de gestos notable en el pelvitrainer, haber practicado repetidas veces en el quirófano experimental la intervención que mejor reproduce el cerdo, como es la nefrectomía, y estar faltos de seguridad al enfrentarnos a esas mismas intervenciones laparoscópicas en el paciente, aunque conozcamos perfectamente los pasos de la misma y tengamos experiencia en su resolución por vía abierta.

Algunos trabajos ponen en tela de juicio la utilidad de estos cursos de pequeña duración en cuanto al seguimiento posterior de una actividad laparoscópica fructífera. El seguimiento de los alumnos que los realizan demuestra que al $5^{\circ}$ año sólo un $8 \%$ refiere que las técnicas laparoscópicas representen más de un 10\% de su actividad quirúrgica ${ }^{8}$.

\section{La tutoria}

El aprendizaje se adquiere con la observación y con la ayuda que se presta a alguien que consideramos experto, maestro en esa materia. Se basa en llegar al conocimiento de una técnica a través de repetición de los pasos de la misma, no sólo en situaciones idóneas, sino también en aquellas que son adversas y que se resuelven con la pericia de alguien con experiencia en su manejo. Más tarde, reproducir esos pasos con la ayuda del maestro en las primeras intervenciones, ser conducido por alguien con mayor experiencia, que nos haga tener confianza en el éxito de la 
intervención y poder avanzar con seguridad en esta etapa de consolidación del aprendizaje.

De esa manera aprendimos en cirugía abierta y así debemos aprender la cirugía laparoscópica y ese debe ser el faro de esta etapa. No creo que los residentes que realicen su formación en servicios con una importante labor laparoscópica precisen pasar por amplias etapas previas de cirugía experimental. Obviamente, el entrenamiento en pelvitrainer será necesario para obtener destreza en la realización de sutura, por ejemplo, pero el residente que participe en intervenciones laparoscópicas, en las que realizará gestos de ayuda que progresivamente serán mayores, desarrollará su aprendizaje mucho más rápidamente.

El entrenamiento clínico de postgraduados por expertos (acreditados después de alcanzar un numero mayor de 50 intervenciones mayores en 2 años) ha demostrado que los alumnos pueden realizar solos una nefrectomía después de haberla realizado menos de 10 veces con el tutor ${ }^{9}$.

Utilizando un tutor en las primeras intervenciones se demuestra que la segunda curva de aprendizaje es sensiblemente menor a la vista de trabajos que analizan del número y gravedad de las complicaciones de una intervención difícil, como es la prostatectomía radical ${ }^{10}$.

Por tanto, creo que el programa que quiere desarrollar la AEU de tutorías realizadas por expertos en laparoscopia será fundamental para potenciar el aprendizaje y progresiva implantación de la laparoscopia en España.

De esta forma, el urólogo aprendiz visitará el centro de trabajo del tutor para asistir de forma activa a un determinado número de cirugías laparoscópicas sobre una determinada técnica y aprender todos aspectos relativos a la misma (preparación del paciente, colocación, instrumentación, pasos quirúrgicos, y cuidados postoperatorios), para después, en una segunda etapa, el tutor se desplace al hospital del alumno para ayudarle y conducirle en la realización de las primeras intervenciones sobre dicha técnica. Existen en España, ya, suficientes expertos para acometer esta tarea que debería iniciarse con técnicas de laparoscopia renal para después conti- nuar por cirugía pélvica (prostatectomía radical, cistectomía radical) de más difícil realización.

Además de la coordinación y soporte económico que debe aportar la AEU, este programa implica la conjunción de esfuerzos de expertos, alumnos, jefes de servicios y gerentes hospitalarios para garantizar su éxito.

Creemos que de esta forma se puede facilitar el aprendizaje, aumentando la confianza y seguridad del alumno en las posibilidades de llevar a buen término las primeras intervenciones, evitando desmoralizadoras reconversiones sin descuidar aspectos relacionados con el compromiso ético con nuestros pacientes.

\section{REFERENCIAS}

1. Amón JH. Creer en la laparoscopia. Arch Esp Urol 2002; 55:347-349.

2. Amón JH. Estado actual de la laparoscopia dentro de la AEU. Boletín informativo AEU 2005;1:17-18.

3. Kaynan AM, Lee K, Winfield HN. Survey of urological laparoscopic practises in the state of California. J Urol. 2002; 167: 2380-2386.

4. Wang DS, Winfield HN. Survey of urological laparoscopic practice patterns in the Midwest. J Urol 2004;172:22822286.

5. Guillonneau B. ¿Should we consider testing for skill in surgery?. Eur Urol. 2005;47:480-481.

6. Shay B.F., Thomas R, Monga M. Urology practice patterns alter residency training in laparoscopy. J Endourol 2002; 16:251-256.

7. Traxer O, Gettman MT, Napper CA, Scott DJ, Jones DB, Roehrborn CG, Pearle MS, Cadeddu JA.The impact of intense laparoscopic skills training on the operative performance of urology residents. J Urol 2001;166(5):1658-1661.

8. Colegrove PM, Winfield HN, Donovan JF Jr, See WA. Laparoscopic practice patterns among North American urologist 5 years after formal training. J Urol. 1999;161(3): 881-886.

9. Shalhav AL, Dabagia MD, Wagner TT, Koch MO, Lingeman JE. Training postgraduate urologists in laparoscopic surgery: the current challenge. J Urol. 2002;167(5):21352137.

10. Fabrizio MD, Tüerk I, Schellhammer PF. Laparoscopic radical prostatectomy: decreasing the learning curve using a mentor initiated approach. J Urol. 2003;169:2063-2065.

Dr. J.H. Amón Sesmero

Hospital del Río Hortega

Cardenal Torquemada, s/n

47010 Valladolid

E-mail: amonsesmero@yahoo.es 